\title{
Dynamic Connectivity between Brain Networks Supports Working Memory: Relationships to Dopamine Release and Schizophrenia
}

\author{
Clifford M. Cassidy, ${ }^{1,2}$ Jared X. Van Snellenberg, ${ }^{1,2}$ - Caridad Benavides, ${ }^{1,2}$ Mark Slifstein, ${ }^{1,2}$ Zhishun Wang, ${ }^{1,2}$ \\ Holly Moore, ${ }^{1,2}$ Anissa Abi-Dargham, ${ }^{1,2}$ and Guillermo Horga ${ }^{1,2}$ \\ ${ }^{1}$ Department of Psychiatry, Columbia University, New York, New York 10032, and ${ }^{2}$ New York State Psychiatric Institute, New York, New York 10032
}

Connectivity between brain networks may adapt flexibly to cognitive demand, a process that could underlie adaptive behaviors and cognitive deficits, such as those observed in neuropsychiatric conditions like schizophrenia. Dopamine signaling is critical for working memory but its influence on internetwork connectivity is relatively unknown. We addressed these questions in healthy humans using functional magnetic resonance imaging (during an $n$-back working-memory task) and positron emission tomography using the radiotracer $\left[{ }^{11} \mathrm{C}\right]$ FLB457 before and after amphetamine to measure the capacity for dopamine release in extrastriatal brain regions. Brain networks were defined by spatial independent component analysis (ICA) and working-memory-load-dependent connectivity between task-relevant pairs of networks was determined via a modified psychophysiological interaction analysis. For most pairs of task-relevant networks, connectivity significantly changed as a function of working-memory load. Moreover, load-dependent changes in connectivity between left and right frontoparietal networks ( $\Delta$ connectivity lFPN-rFPN) predicted interindividual differences in task performance more accurately than other fMRI and PET imaging measures. $\Delta$ Connectivity lFPN-rFPN was not related to cortical dopamine release capacity. A second study in unmedicated patients with schizophrenia showed no abnormalities in load-dependent connectivity but showed a weaker relationship between $\Delta$ connectivity lFPN-rFPN and working memory performance in patients compared with matched healthy individuals. Poor working memory performance in patients was, in contrast, related to deficient cortical dopamine release. Our findings indicate that interactions between brain networks dynamically adapt to fluctuating environmental demands. These dynamic adaptations underlie successful working memory performance in healthy individuals and are not well predicted by amphetamineinduced dopamine release capacity.

Key words: dopamine; multimodal imaging; network functional connectivity; positron emission tomography; schizophrenia; working memory

\section{Significance statement}

It is unclear how communication between brain networks responds to changing environmental demands during complex cognitive processes. Also, unknown in regard to these network dynamics is the role of neuromodulators, such as dopamine, and whether their dysregulation could underlie cognitive deficits in neuropsychiatric illness. We found that connectivity between brain networks changes with working-memory load and greater increases predict better working memory performance; however, it was not related to capacity for dopamine release in the cortex. Patients with schizophrenia did show dynamic internetwork connectivity; however, this was more weakly associated with successful performance in patients compared with healthy individuals. Our findings indicate that dynamic interactions between brain networks may support the type of flexible adaptations essential to goal-directed behavior.

\section{Introduction}

Complex cognitive processes are thought to arise from orchestrated communication at the level of brain networks. Research in

Received Sept. 2, 2015; revised Feb. 29, 2016; accepted March 4, 2016.

Author contributions: C.M.C., J.X.V.S., M.S., H.M., A.A.-D., and G.H. designed research; C.M.C., J.X.V.S., M.S., A.A.-D., and G.H. performed research; C.M.C., C.B., M.S., Z.W., A.A.-D., and G.H. analyzed data; C.M.C., C.B., and G.H. wrote the paper. the last decade has uncovered an intrinsic organization of the brain into functional networks that operate partly in parallel during rest and are thought to interact during complex cognitive
This work was supported by Grant 5P50MH086404-05 from the National Institute of Mental Health and the Sylvio 0. Conte Center for the Study of Dopamine Dysfunction in Schizophrenia and a grant from the Sidney R. Baer Jr Foundation; C.M.C. was supported by a postdoctoral fellowship from the Fonds de Recherche du Québec, Santé; G.H. was supported by a Research Career Development Award from the National Institute of Mental Health, 1 K23 MH101637. 
processes such as working memory (Fox et al., 2005; Linden, 2007; Parnaudeau et al., 2013; Shen et al., 2015), an interaction that may afford maintenance and manipulation of perceptual information toward goal-directed behavior. These recent advances are beginning to elucidate cognition at a systems level, yet it remains largely unknown how brain networks interact to adapt to changing cognitive demands such as increased workingmemory load. Understanding these mechanisms could provide insight into the basis of interindividual differences in working memory performance, often unexplained or unreported in studies using conventional fMRI measures (Balsters et al., 2013; Saliasi et al., 2014), and clarify the perplexing nature of working memory deficits in schizophrenia (Lee and Park, 2005).

Functional-connectivity networks are sets of spatially distributed brain regions with temporally coordinated activity (Damoiseaux et al., 2006) that can be ascertained with multivariate analyses of $\mathrm{fMRI}$, such as independent component analysis (ICA). Some activate during cognitive tasks, dubbed "taskpositive" or "control" networks, including the frontoparietal network (FPN) and the cingulo-opercular network (CON; or "salience" network), whereas others such as the default-mode network (DMN) activate at rest and deactivate during various tasks ("task-negative" networks; Fox et al., 2005). Functional connectivity is often studied during resting state as anchanging feature that remains stationary throughout an MRI scan (referred to as static, intrinsic, or task-independent connectivity; Damoiseaux et al., 2006). In contrast to resting-state studies, task-based studies provide a built-in structure allowing the investigation of variability in functional connectivity in relation to controlled task demands, a type of dynamic- or task-dependent connectivity (Rasetti et al., 2011; Hutchison et al., 2013; Bray et al., 2015). Here, we used an established working-memory paradigm, the $n$-back task, to investigate interactions of brain networks that may represent systems-level adjustments to varying working-memory loads.

Dopamine is a key neuromodulator that may influence interactions between brain networks relevant for cognition. Cortical dopamine plays an important role in working memory (Sawaguchi and Goldman-Rakic, 1991; Cools and D'Esposito, 2011; Slifstein et al., 2015), where it promotes stability of working memory networks by increasing their signal-to-noise ratio (Rolls et al., 2008), which may in turn modulate functional connectivity in the context of working memory (Sambataro et al., 2009). Manipulations that increase (Kelly et al., 2009; Cole et al., 2013) or decrease (Nagano-Saito et al., 2008) dopamine signaling have indeed been shown to alter functional connectivity between individual brain regions. The only study evaluating the role of dopamine on connectivity between networks found that dopamine synthesis capacity in the midbrain correlated positively with functional connectivity between the FPN and DMN at rest (Dang et al., 2012); however, the role of cortical dopamine in modulating systems-level interactions across brain networks subserving human cognition has not been studied.

The main objective of the current work was to test whether interactions between brain networks play a significant role in adaptive cognitive processes and whether these network interac-

The authors declare no competing financial interests.

Correspondence should be addressed to either Dr. Guillermo Horga or Dr. Clifford Cassidy, Department of Psychiatry, New York State Psychiatric Institute, Columbia University Medical Center, 1051 Riverside Drive, New York, NY 10032. E-mail: HorgaG@nyspi.columbia.edu or Cassidy@nyspi.columbia.edu.

DOI:10.1523/JNEUROSCI.3296-15.2016

Copyright $\odot 2016$ the authors $\quad 0270-6474 / 16 / 364378-12 \$ 15.00 / 0$ tions depend on cortical dopamine release capacity as measured by $\left[{ }^{11} \mathrm{C}\right]$ FLB457 PET combined with the amphetamine paradigm (Slifstein et al., 2015; Study 1). After defining functional connectivity networks using spatial ICA on BOLD activity, we investigated changes in connectivity between pairs of task-relevant networks as a function of working-memory load (henceforth referred to as load-dependent connectivity). Our primary hypotheses were that: (1) with increasing working memory load, the connectivity between task-positive networks would increase, whereas the connectivity between task-positive and DMN would decrease; (2) this load-dependent internetwork connectivity would predict interindividual differences in task performance; and (3) would correlate with cortical dopamine release capacity. Study 2 evaluated the relevance of load-dependent internetwork connectivity to neuropsychiatric illness, by investigating its relationship to task performance and clinical severity in unmedicated patients with schizophrenia.

\section{Materials and Methods Study 1 (healthy individuals) \\ Participants}

Forty-three healthy individuals (19 male) participated in this study, which was approved by the Institutional Review Boards of the New York State Psychiatric Institute (NYSPI) and the Yale University Human Investigation Committee. This study was part of a larger investigation including PET and fMRI. Although the PET data have been previously published, (Slifstein et al., 2015), none of the $n$-back fMRI data have been published, nor have the relationships between the two sets of measures. All participants provided written informed consent and were recruited through advertisements. Inclusion criteria were as follows: absence of any current or past DSM-IV Axis-I diagnosis (determined by an abbreviated version of the Structured Clinical Interview for DSM-IV Axis I disorders; First et al., 1995), and no family history (first-degree) of psychotic illness. Exclusion criteria were as follows: significant medical and neurological illnesses, current misuse of substances other than nicotine, positive urine drug screen, pregnancy, and nursing. For information on participant characteristics see Table 1.

Some participants were excluded postscanning based on the quality of their data. To ensure all participants retained in analysis performed the behavioral task as instructed, participants were excluded if their performance on the less challenging (1-back) condition was not significantly above chance ( $p<0.05$; single-subject binomial test); one participant was excluded due to this criterion. Three additional participants were excluded due to poor fMRI data quality (see fMRI preprocessing); leaving a sample of 39 participants (18 male) with usable fMRI data, of which 15 also had usable PET data. Participant and parental socioeconomic status were calculated according to the Hollingshead scale (Hollingshead, 1975); handedness was measured with the Edinburgh Handedness Inventory (Oldfield, 1971).

\section{Task procedures}

The $n$-back working-memory task tests the ability to recognize repetitions in a series of consecutively presented stimuli. The working-memory load of the task is varied by increasing the interval at which repetitions must be identified. We used a 1-back condition in which participants must identify if each stimulus matches the one immediately preceding it and a more challenging 2-back condition in which participants must identify if each stimulus matches the one preceding it by 2 stimuli. Our version of the task (Fig. 1A) used visual stimuli consisting of simple line drawings of $3 \mathrm{D}$ objects (the same stimuli have been used previously in different tasks (Curtis et al., 2000; Van Snellenberg et al., 2015) and two-alternative forced-choice responses whereby participants pressed one key in a two-button response device to identify targets and the other key to identify nontargets (the left vs right button were pressed to identify targets vs nontargets in a counterbalanced order across participants).

Each participant performed eight blocks of the 1-back condition and eight blocks of the 2-back condition with each block comprising 10 stim- 
Table 1. Demographic, behavioral, and clinical measures

\begin{tabular}{|c|c|c|c|c|c|}
\hline & $\begin{array}{l}\text { All healthy controls, } \\
N=39\end{array}$ & $\begin{array}{l}\text { Matched healthy } \\
\text { controls, } N=23\end{array}$ & $\begin{array}{l}\text { Patients with } \\
\text { schizophrenia, } N=15\end{array}$ & $\begin{array}{l}p \text { value all healthy } \\
\text { controls-schizophrenia }\end{array}$ & $\begin{array}{l}p \text { value matched healthy } \\
\text { controls-schizophrenia }\end{array}$ \\
\hline Male gender & $18(46 \%)$ & $11(48 \%)$ & $8(53 \%)$ & 0.43 & 1.0 \\
\hline Age $\pm S D$ & $33.5 \pm 8.2$ & $36.0 \pm 8.3$ & $35.1 \pm 11.0$ & 0.56 & 0.79 \\
\hline African American & $14(36 \%)$ & $12(52 \%)$ & $8(53 \%)$ & 0.20 & 1.0 \\
\hline Right-handed & $38(97 \%)$ & $22(96 \%)$ & $13(87 \%)$ & 0.18 & 0.55 \\
\hline Removed fMRI volumes & $59.2 \pm 76.2$ & $84.5 \pm 89.5$ & $95.2 \pm 74.6$ & 0.12 & 0.70 \\
\hline Positive symptoms (SAPS global total score) & - & - & $4.6 \pm 2.8$ & - & - \\
\hline Negative symptoms (SANS global total score) & - & - & $8.1 \pm 5.1$ & - & - \\
\hline Duration of illness (years) & - & - & $16.8 \pm 11.2$ & - & - \\
\hline
\end{tabular}

A

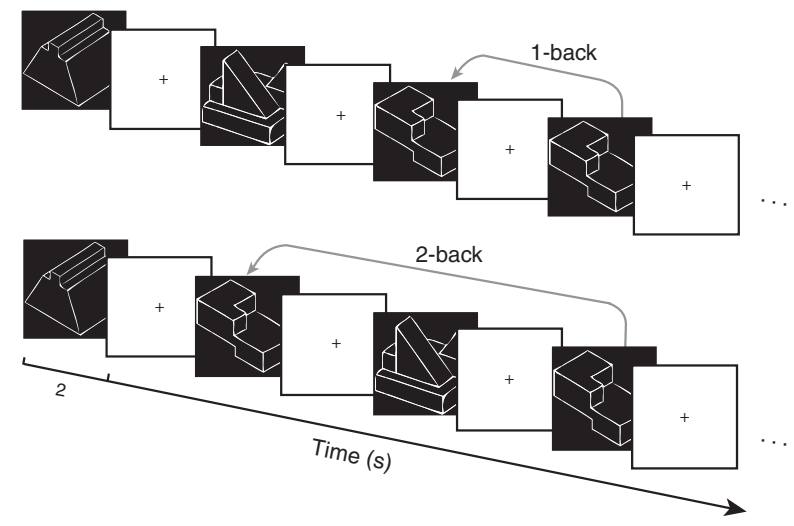

B

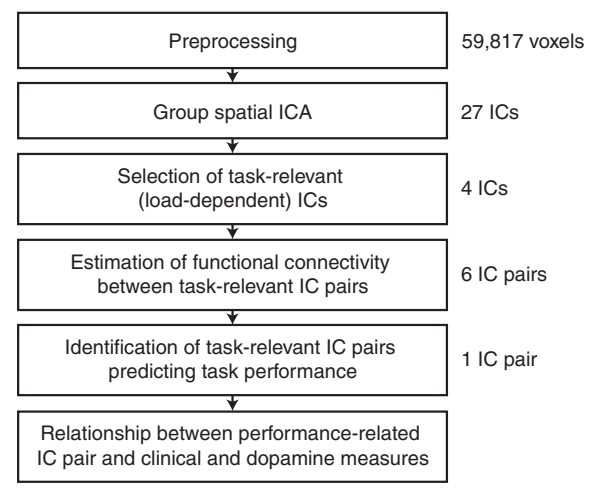

C

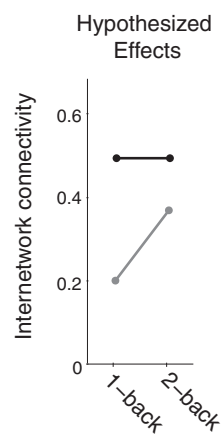

Figure 1. $\quad A$, Structure of the $n$-back task. Participants must identify matching stimuli presented in succession (1-back condition) or separated by an intervening stimulus (2-back condition). Ten stimuli were presented per block and there were 8 blocks of each condition. Stimuli are represented in the figure at a disproportionate scale and did not extend to the periphery of the screen. $\boldsymbol{B}$, Schematic of the fMRI analysis pipeline. Each stage of the fMRI analysis is depicted together with the number of elements of fMRI data produced at that stage. The first two steps of analysis included healthy participants and schizophrenia patients together; all later stages of analysis were performed in the two groups separately. C, Schematic illustrating load-dependent internetwork connectivity. The black line represents a hypothetical pair of networks that has high load-independent connectivity (has high connectivity of $\sim 0.5$ points at both the 1-back and 2-back conditions) but near-zero load-dependent connectivity (no change in connectivity from the 1-back to the 2-back condition). The gray line represents a different hypothetical pair of networks showing high load-dependent connectivity (an increase in connectivity of $\sim 0.2$ points between the 1-back and the 2-back conditions) but lower load-independent connectivity than the black pair.

uli (9 trials for 1-back, 8 trials for 2-back). Each stimulus presentation lasted $2 \mathrm{~s}$ and was followed by $2 \mathrm{~s}$ during which a fixation cross appeared before the next stimulus presentation. Task performance was calculated using adjusted hit rate (AHR; the percentage of properly identified targets corrected for false-positives) as described previously (MacMillan, 2005; Abi-Dargham et al., 2012). This metric ranges from a maximum possible score of 1 for perfect performance to -1 if all true targets are missed and all non-targets are incorrectly identified as targets. Conventional indices of performance on an $n$-back task include accuracy, $\mathrm{d}^{\prime}$, and AHR (MacMillan, 2005). These can be used as absolute measures of performance on one condition or differential performance between conditions (2-back performance - 1-back performance). AHR is commonly used to index $n$-back performance (Abi-Dargham et al., 2012; Narendran et al., 2012; Slifstein et al., 2015; Terán-Pérez et al., 2012) and we selected AHR during the 2-back condition as our primary measure of interest. This measure was highly correlated with the other five performance measures (differential measures of AHR, accuracy, and d', as well as absolute measures of accuracy and d'; Pearson $r$ values ranged from 0.55 to 0.96 ). Because the $n$-back is a signal detection task with a built-in workingmemory burden we preferred a measure derived from signal detection theory, such as d' or AHR. Of those two, although 2-back d' is commonly used and has been shown to have maximal sensitivity to detect workingmemory deficits in schizophrenia (Haatveit et al., 2010), we preferred to use AHR since this measure does not transform the signal and noise measures, a transformation that may not be desirable as it can introduce a bias favoring aggressive strategies. The two measures are nonetheless similar and were very highly correlated (Pearson $r$ between the 2 measures $=0.96$ ). Furthermore, we preferred an absolute over a differential measure of performance because differential measures are not able to accurately measure change in performance from 1-back to 2-back in the case of participants who are not performing well above chance in the less challenging, 1-back, condition. We have previously used the absolute AHR measure of working memory performance in the $n$-back for these reasons (Abi-Dargham et al., 2012; Slifstein et al., 2015). Nonetheless, to ensure the findings were generalizable across measures, we also examined another widely used measure, differential accuracy (2-back - 1-back) operationalized as percentage correct responses on the 2-back minus percentage correct responses on the 1-back; the performance measure that differed most from 2-back AHR quantitatively and qualitatively.

\section{fMRI methods}

fMRI data acquisition. Imaging was performed on a Philips $1.5 \mathrm{~T}$ Intera scanner at the Columbia Radiology MRI Center at the Neurological Institute of New York. T1-weighted images were obtained with a spoiled gradient recalled sequence (field-of-view $=256 \mathrm{~mm}, 200$ slices, $1 \mathrm{~mm}$ isotropic voxels). Whole-brain functional echo-planar images (EPIs) were obtained using an 8-channel SENSE coil (SENSE factor of 1.5, TR = $2 \mathrm{~s}, \mathrm{TE}=28 \mathrm{~ms}$, flip angle $=77^{\circ}$, field-of-view $=192 \mathrm{~mm}, 40$ slices, $3 \mathrm{~mm}$ isotropic voxels). Participants completed four runs of 145 volumes, each of which included four $40 \mathrm{~s}$ blocks (2 blocks of 1-back and 2 of 2-back), for a total scanning time of $19 \mathrm{~min}$ and $20 \mathrm{~s}$. Twenty-six seconds of rest occurred between blocks, for $6 \mathrm{~s}$ of which task instructions were pre- 
A

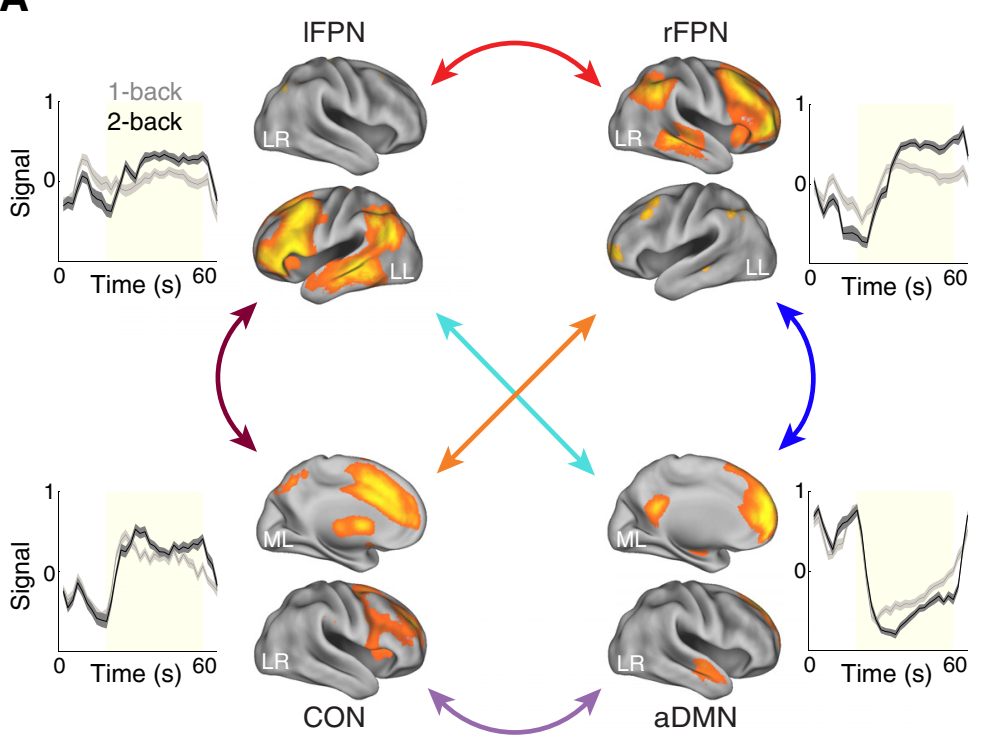

B

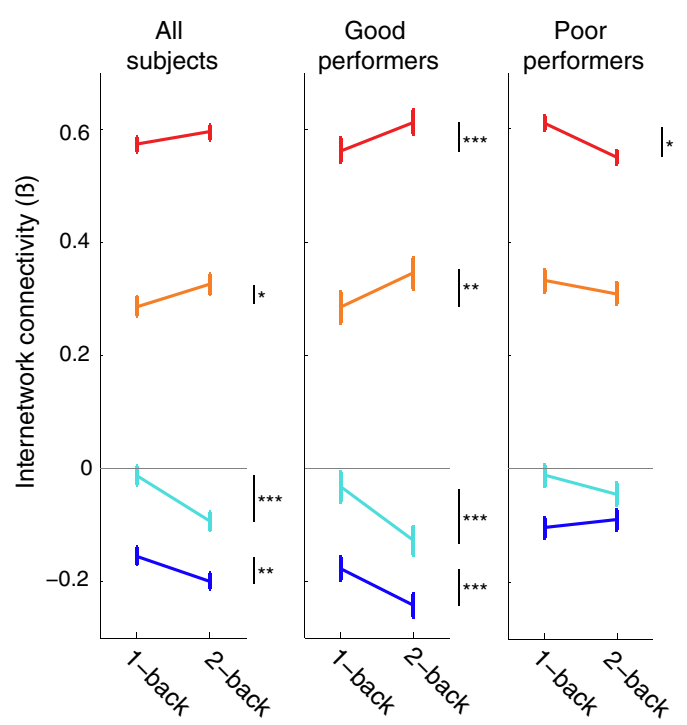

IFPN-rFPN rFPN-CON IFPN-aDMN rFPN-aDMN IFPN-CON CON-aDMN

Figure 2. A, Functional connectivity networks involved in working memory in healthy individuals. Displayed are 3D-rendered spatial maps of $4 \mathrm{ICA}$ components (thresholded at $t>5.0$ in orange and ranging up to $t>15.0$ in yellow) along with plots of their time-locked average time courses. Components were deemed to represent working memory networks based on differential activity in the 2-back and 1-back conditions as illustrated by the plots. The plotted lines represent activity of the component averaged over blocks and subjects and bounded by the SEM (shaded area); the yellow box on the plots represents the time between task onset and offset. Connectivity between pairs of these networks is illustrated by color-coded arrows with connectivity between task-positive networks represented by warm colors and connectivity between the task-negative aDMN and task-positive networks represented by cold colors. $\boldsymbol{B}$, Load-dependent changes in internetwork connectivity. Connectivity between pairs of networks (indexed by the standardized $\beta$ value) is plotted in the 1-back and 2-back conditions for the four network pairs showing greatest loaddependent connectivity using the color codes indicated in Figure $2 A$. Separate plots present data from all participants $(n=39)$, good performers $(n=29)$, and poor performers $(n=10)$. For good performers, connectivity significantly changed with cognitive load for all four pairs shown. Error bars are $95 \%$ confidence intervals representing within-subjects variation (Cousineau, 2005). LR, Lateral right; LL, lateral left; ML, medial left. One-sample $t$ test. ${ }^{* * *} p<0.001,{ }^{* *} p<0.01,{ }^{*} p<0.05$.

sented. Behavioral responses were registered with a trackball response device.

fMRI preprocessing. Standard preprocessing procedures were implemented using SPM8 (http://www.fil.ion.ucl.ac.uk/spm/) and custom scripts in MATLAB 2013b. Slice-timing correction was implemented using SPM8 and motion realignment using INRIAlign (Freire et al., 2002). T1 and EPI images were then manually realigned to provide better starting estimates for coregistration. Six-parameter affine coregistration was used to coregister the functional runs to each other and to the individual participants' $\mathrm{T} 1$ image, and participants' $\mathrm{T} 1$ images and all functional images were then coregistered to the International Consortium for Brain Mapping template. Next, T1 structural images were segmented into three tissue compartments and the spatial normalization parameters from the segmentation algorithm were applied to the coregistered $\mathrm{T} 1$ and all EPI images. EPI images were then smoothed using an $8 \mathrm{~mm}^{3}$ fullwidth-at-half-maximum Gaussian kernel. In keeping with recent guidelines for functional connectivity analysis (Power et al., 2012), we next removed volumes ("scrubbed") by eliminating those showing excessive motion or signal spikes [framewise displacement and DVARS thresholds were based on the median plus 2 interquartile ranges for a larger imaging dataset including schizophrenia patients and healthy individuals (1.11 and 38.3, respectively); volumes exceeding either limit were removed together with the 1 prior and 2 succeeding volumes]. If $>50 \%$ of volumes in a run were removed, the entire run was excluded. After eliminating volumes and runs in this manner, participants who did not retain a cumulative total of at least 300 of the 580 volumes (did not retain $10 \mathrm{~min}$ of usable data) were excluded. Three healthy participants were retained despite having one of their four runs eliminated.

$f M R I$ data analysis. Figure $1 B$ depicts the fMRI data analysis pipeline. A group spatial ICA was performed by standard methods using the infomax algorithm (Bell and Sejnowski, 1995) in the group ICA of Functional MRI Toolbox (http://mialab.mrn.org). Before ICA, the optimal number of independent components was first determined using a modified min- imum length algorithm (Li et al., 2007). ICA defines independent components (ICs) reflecting spatially independent and temporally coherent networks. This process uses information from the entire group of participants to produce, for each IC, a spatial map with the weights for each voxel's contribution to that IC and a time course reflecting the temporal pattern of activity in that IC. Time courses and spatial maps for each participant's set of components can then be back reconstructed. To display spatial maps of ICs (Fig. 2A), one-sample $t$ tests were performed incorporating all individual subject maps and then $t$ values were rendered onto a standard cortical surface using Caret 5 software. In performing ICA analysis, data from healthy controls and patients with schizophrenia (the latter group from Study 2; see below) were combined. It has been previously shown that performing ICA on all participants does not alter the results compared with performing ICA on individual groups separately (Calhoun et al., 2008) but has the benefit of matching components across groups to allow for between-groups analysis. Aside from the inclusion of patients in defining the ICA components, all analyses in Study 1 were run only on healthy controls. When including only healthy controls to define the ICA components, the load-dependent connectivity measures (our primary measures of interest) were highly correlated to those produced from the ICA including patients and controls (Pearson $r$ ranged from 0.98 to 0.99 for the 6 network pairs discussed below). Following ICA, the IC time courses were further processed in MATLAB before final analyses: removed (scrubbed) volumes were replaced using linear interpolation, the time courses were broken into runs, high-pass filtered (cutoff $=0.005 \mathrm{~Hz}$ ), de-spiked (time points with Cook's distance $>4 /$ number of time points were replaced with the average of the preceding and succeeding time points), and Z-scored within each run.

To select networks of interest for subsequent functional connectivity analyses we first removed components likely to be spurious (ie, related to head movement, ventricle activity, or other signal artifacts) based on their spatial overlap with white matter and/or CSF (6 ICs were removed following consensus between 2 independent raters; the remaining 21 ICs 
thus represented putative brain networks). We then determined the relationship between the activity of the remaining independent components and the working-memory task by performing a subject-level linear regression on each of the IC time courses. The design matrix included as regressors (1-2) two boxcar functions defined by the onsets and durations of the 1-back and 2-back blocks, respectively, and also nuisance regressors: [(3) a boxcar function corresponding to the presentation of the instruction screen, $(4,5)$ impulse functions indicating left and right button presses, (6-29) 6 motion parameters as well as the derivative, square, and squared derivative of each, and additionally (30+) 1 dummy regressor for every scrubbed volume]. For each of the nonspurious ICs and each participant, the regression coefficients or $\beta$ values for the 2-back condition were subtracted from those for the 1-back condition to obtain a contrast estimate of the change in activation with working-memory load. Several ICs showed significant change in activation with workingmemory load based on a (group-level) one-sample $t$ test of the contrast estimates (2-back - 1-back), indicating that they reflected workingmemory-load-related networks; of these four were retained for functional connectivity analyses (see Results for justification for this selection). These four networks showed moderate to strong spatial correlation (Pearson $r$ values between 0.34 and 0.55 ) with T-maps of canonical representations of corresponding default-mode, frontal, and attentional networks derived from resting-state scans of a large dataset of healthy participants (Allen et al., 2011; http://mialab.mrn.org/data/hcp/ RSN_HC_unthresholded_tmaps.nii).

Finally, internetwork functional connectivity analyses were performed on the scrubbed time courses of these four ICs. We used Fisher-transformed Pearson correlation coefficients between all six pairs of network time courses to create an index of intrinsic connectivity averaged throughout the experiment which we refer to as load-independent connectivity. To index changes in connectivity with working-memory load, which we refer to as load-dependent connectivity, our focus in the current study, linear regression analyses were performed with one IC time course as outcome variable (Network A) and as regressors the other IC time course (Network B), the block index variable (1-back vs 2-back), and the interaction variable (block $\times$ Network B), in the vein of psychophysiological interaction analyses (Friston et al., 1997) with regressors $Z$-scored. The $\beta$ value of the interaction term provided an index of loaddependent connectivity. The block-index variable was coded with zeros for 1-back task blocks and associated rest/preparatory periods and ones for 2-back task blocks and associated rest/preparatory periods. Including periods of active task and rest/preparatory periods together for the analysis of connectivity has been recommended (Hutchison et al., 2013 ) in analyzing load-dependent connectivity to avoid neglecting important connectivity dynamics occurring in preparation of the upcoming task (Eichele et al., 2008; Thompson et al., 2013). Furthermore, this strategy may allow more robust measurement of connectivity by capturing a substantial source of variance in the time courses as networks activate and deactivate through the course of engaging in and disengaging from the task. We assigned the ICs to be the independent or dependent variable based on the order in which, on average, they tended to activate when engaging in working memory with the later activating IC being assigned as the dependent variable. In pairs including a task-negative IC, the task-positive IC was always set as the dependent variable.

\section{PET study design and data analysis}

Complete description of PET acquisition and analysis were previously published (Slifstein et al., 2015). A subset of 15 of the healthy controls (8 male) had usable fMRI, behavioral, and PET data. Participants underwent two PET scans on $1 \mathrm{~d}$ with the radiotracer $\left[{ }^{11} \mathrm{C}\right] \mathrm{FLB} 457$ at the Yale University PET Center. A 90 min baseline scan was acquired, followed immediately by oral administration of amphetamine $(0.5 \mathrm{mg} / \mathrm{kg})$ and a second 90 min scan was acquired $3 \mathrm{~h}$ after amphetamine administration. Arterial plasma data were collected to form metabolite-corrected input functions. Data were acquired on an HR+ scanner (Siemens). Regionsof-interest (ROIs) were drawn on each participant's high resolution T1-weighted MRI scan according to previously described criteria (Abi-Dargham et al., 2000, 2012). These ROIs included cortical and sub- cortical, extrastriatal regions; for the current study the ROI analyzed was the DLPFC given the critical role of this brain region in working memory (Weinberger et al., 1988; Sawaguchi and Goldman-Rakic, 1991; Durstewitz and Seamans, 2008; Rolls et al., 2008;Arnsten and Jin, 2014). Data were analyzed with a two-tissue compartment model that incorporated a set of shared parameter estimates across regions. Binding potentials with respect to the nondisplaceable compartment $\left(\mathrm{BP}_{\mathrm{ND}}\right)$ were estimated. We computed the relative change in $\mathrm{BP}_{\mathrm{ND}}$ following amphetamine $\left(\Delta \mathrm{BP}_{\mathrm{ND}}\right)$ in each ROI according to $\Delta \mathrm{BP}_{\mathrm{ND}}=\mathrm{BP}_{\mathrm{ND}}$ (postamphetamine $/ \mathrm{BP}_{\mathrm{ND}}$ (baseline) -1 .

Behavioral, fMRI functional connectivity, and PET measures were related to each other using linear regression analyses (Baron and Kenny, 1986; Wager et al., 2009).

\section{Study 2 (healthy individuals versus patients with schizophrenia)}

Participants and clinical measures

A subset of 23 of the healthy participants from Study 1 (11 male) were selected for Study 2 (Table 1). This subsample was selected to improve matching as there was evidence that the full sample differed from the schizophrenia sample on working memory performance, fMRI data quality, handedness, and race (Table 1). All results were nonetheless similar when comparing patients with schizophrenia to either the full sample or this subsample of healthy controls. Twenty unmedicated patients with schizophrenia (11 male) also participated in this study. Patients were recruited from the inpatient and outpatient research facilities at NYSPI and an assessment of capacity was performed by an independent psychiatrist before providing consent. Inclusion criteria for patients were as follows: (1) lifetime DSM-IV diagnosis of schizophrenia, schizoaffective or schizophreniform disorder; (2) no bipolar disorder; (3) no antipsychotics for 3 weeks before PET scan; and (4) no history of violent behavior. Exclusion criteria were similar to those for healthy controls. Five patients with schizophrenia were excluded due to poor task performance and/or poor quality fMRI data according to the same criteria used in Study 1, leaving a final sample of 15 patients ( 8 male), 10 of whom ( 5 male) had PET data. Three patients were retained despite having one run of fMRI data eliminated. Diagnostic status was determined with the Diagnostic Interview for Genetic Studies (Nurnberger et al., 1994) followed by a consensus diagnosis conference. Severity of symptoms was assessed using total global scores from the Scale for the Assessment of Positive symptoms (SAPS; Andreasen, 1984) and the Scale for Assessment of Negative Symptoms (SANS; Andreasen, 1983). Clinical assessments were administered by trained interviewers. Further clinical and demographic information is in Table 1.

\section{fMRI and PET methods}

The same procedures were used as in Study 1 for the behavioral task, and also for the acquisition and analysis of fMRI and PET data. Loaddependent connectivity measures were compared between healthy controls and schizophrenia groups using two-sample $t$ tests. The independent functional spatial maps (intranetwork connectivity maps representing mixing coefficients of the components for each voxel as derived from ICA; Fig. 2A) for the four networks of interest were compared between the groups in a control analysis using voxelwise, univariate twosample $t$ tests to identify voxels showing differential weights on a given IC (voxels showing differential intranetwork connection strengths) in controls versus patients, which would indicate that a given network's spatial structure differs between the groups. Behavioral, fMRI functional connectivity, PET, and clinical measures were related to each other using linear regression analyses.

\section{Results}

\section{Study 1 (healthy individuals)}

Behavioral performance

Working memory performance decreased from the 1-back $($ mean \pm SD: $\mathrm{AHR}=0.90 \pm 0.12$; accuracy $=94 \pm 7.6 \%)$ to the 2-back condition $(\mathrm{AHR}=0.76 \pm 0.21$; accuracy $=86 \pm 14 \%)$. This decrease was statistically significant (AHR: $t_{(38)}=-6.1$, 
Table 2. Connectivity between pairs of networks in healthy individuals $(n=39)$

\begin{tabular}{lcccc}
\hline & IFPN & \multicolumn{1}{c}{ rFPN } & CON & \multicolumn{1}{l}{ aDMN } \\
\hline IFPN & & $0.05^{* * *}$ & $0.03^{*}$ & $-0.10^{* * *}$ \\
rFPN & $0.57^{* * *}$ & & $0.06^{* *}$ & $-0.07^{* * *}$ \\
CON & $0.30^{* * *}$ & $0.29^{* * *}$ & & -0.01 \\
aDMN & -0.02 & $-0.16^{* * *}$ & $-0.35^{* * *}$ & \\
\hline
\end{tabular}

Load-independent connectivity (bottom left, shaded; values are Fisher-transformed Pearson correlation coefficients averaged across all subjects) and load-dependent connectivity (top right, values are the change in connectivity from 1 -back to 2-back averaged across good performers).

One-sample $t$ test. ${ }^{* * *} p<0.001,{ }^{* *} p<0.01,{ }^{*} p<0.05$.

$p=0.0000004$; accuracy: $\left.t_{(38)}=-5.0, p=0.00002\right)$. Only 10 participants $(26 \%)$ and 3 participants $(8 \%)$ made no errors in the 1-back and the 2-back conditions, respectively, indicating that this object-based version of the task was more challenging than more common letter variants.

\section{Identification of functional connectivity networks involved in working memory}

To define functional connectivity networks we performed group spatial ICA on preprocessed BOLD signal time courses. The optimal number of spatial ICs was estimated before ICA analysis to be 27 (median across subjects $=26.5$, mean across subjects $=$ 26.7). The ICA components of interest were selected by first removing artifactual components and then identifying which of the remaining components, representing putative brain networks, were involved in working memory by independently regressing all IC time courses against a model including task-related and nuisance regressors (see fMRI Methods). To identify working memory (WM)-related networks (for the purpose of data reduction and selection rather than hypothesis testing per se), we searched for ICs in which there was a significant load-dependent activation. In the 2-back minus 1-back contrast, three networks showed increased activation (WM-task-positive) and 6 networks showed decreased activation (WM-task-negative; significant one-sample $t$ test). We retained as networks of interest all $3 \mathrm{WM}$ task-positive ICs: a left FPN (IFPN; $\left.t_{(38)}=4.3 ; p=0.0001\right)$, a right FPN $\left(\mathrm{rFPN} ; t_{(38)}=4.7 ; p=0.00,003\right)$ and the CON $\left(t_{(38)}=\right.$ $3.3 ; p=0.002$; Fig. $2 A)$. Of the six WM-task-negative networks, we only retained the anterior DMN (aDMN; Fig. $2 A$ ) given our a priori hypothesis and to minimize the number of tests. Notably, the aDMN showed the greatest working-memory-dependentdeactivation $\left(t_{(38)}=-5.3 ; p=0.000,006\right)$; the posterior DMN was not retained because it showed similar deactivation in the 1 -back and 2-back conditions $\left(t_{(38)}=0.67 ; p=0.51\right)$. The spatial maps of these four networks were highly consistent with canonical ICA-derived networks (Damoiseaux et al., 2006; Fig. 2A).

\section{Load-dependent changes in internetwork connectivity}

We performed internetwork functional connectivity analysis on all six possible pairs of the four ICs retained. Pearson correlation coefficients (Fisher $z$-transformed) between pairs of IC time courses over the full experiment were calculated to provide a measure of load-independent connectivity similar to that conventionally reported in the functional connectivity literature. We found that the time courses of the three WM-task-positive ICs were positively correlated with each other and negatively correlated with the time series of the WM-task-negative aDMN (Table 2). We next investigated load-dependent connectivity by testing a psychophysiological interaction indicating the strengthening or weakening of connectivity between networks as a function of working-memory load (schematically illustrated in Fig. 1C). The analysis showed a significant load-dependent change in connectivity for three of the six network pairs (onesample $t$ test of standardized interaction $\beta$-values; rFPN-CON, $p=0.029 ; \mathrm{rFPN}-\mathrm{aDMN}, p=0.005 ; \mathrm{lFPN}-\mathrm{aDMN}, p=0.00,002$; Fig. $2 B$ ), indicating a significant change in connectivity with working-memory load. We performed a further analysis in which we excluded participants in the lowest quartile of performance on the 2-back condition because we observed that load-dependent connectivity in these participants showed an opposite trend compared with good performers (Fig. 2B). The remaining 29 good performers showed significant load-dependent connectivity for five of the six pairs (one-sample $t$ test of standardized interaction $\beta$-values; Table 2; Fig. $2 B$ ).

\section{Behavioral correlates of load-dependent internetwork connectivity}

We next sought to determine the relationship between loaddependent connectivity and performance in the working-memory task. We used a backward stepwise linear regression with working memory performance as the outcome variable and load-dependent connectivity between all six pairs of ICs as regressors. This allowed us to see which of the pairs contributed independently to the prediction of working memory performance by successively removing the least informative pair until only significant $(p<0.05)$ predictors remained. With either behavioral measure as the dependent variable, we found only one predictor was retained in the final model: loaddependent connectivity between IFPN and rFPN [referred to as $\Delta$ connectivity lFPN-rFPN; 2-back AHR: $R^{2}=0.30$, standardized $\beta=$ $0.55, p=0.0003$; differential accuracy (2-back - 1-back): $R^{2}=0.15$, standardized $\beta=0.39, p=0.014$; Table 3; Fig. $3 A]$, indicating that an increase in connectivity between IFPN and rFPN with increasing WM load predicted better task performance across subjects.

We next wanted to investigate whether load-dependent connectivity would be the best predictor of task performance compared with more commonly used measures, such as loadindependent connectivity, load-dependent activation, or PET dopamine measures. We used the same load-independent connectivity and load-dependent activation measures described above, as well as DLPFC D2 receptor availability $\left(\mathrm{BP}_{\mathrm{ND}}\right.$ of the radiotracer $\left.\left[{ }^{11} \mathrm{C}\right] \mathrm{FLB} 457\right)$ and DLPFC dopamine release capacity $\left(\triangle B P_{N D}\right.$ before and after amphetamine administration). For each type of measure, we performed backward stepwise regressions to predict performance. Regression models including load-independent connectivity between the six IC pairs and regressions including PET dopamine measures did not retain any significant predictors of either behavioral measure (Table 3; Fig. 3). Regression models including load-dependent activation of the four ICs did not retain any networks as predictors of differential accuracy (2-back 1 -back) but did retain activation of the CON and IFPN as predictors of 2-back AHR $\left(R^{2}=0.22 ; \mathrm{CON}\right.$ : standardized $\beta=$ $-0.47, p=0.012$; lFPN: standardized $\beta=0.52, p=0.005$; Table 3; Fig. 3). However, multiple lines of evidence indicated that the load-dependent connectivity model was superior to the load-dependent activation model in explaining working memory performance. First, a direct model comparison indicated that the load-dependent connectivity model retained more information than any of the other models and this was true for both working memory metrics (Comparing Akaike Information Criterion across models showed probabilities $<5 \%$ that any other model was superior to the loaddependent connectivity model; Table 3; Fig. 3). Second, a backward stepwise linear regression predicting 2-back AHR based on load-dependent activation in IFPN and CON, as well as 
Table 3. Backward stepwise regressions to predict working-memory performance using different types of fMRI measures in healthy individuals

\begin{tabular}{|c|c|c|c|c|c|c|c|c|}
\hline \multirow[b]{2}{*}{$\begin{array}{l}\text { Task performance measure } \\
\text { (dependent variable) }\end{array}$} & \multirow[b]{2}{*}{$\begin{array}{l}\text { Type of imaging } \\
\text { measure }\end{array}$} & \multirow[b]{2}{*}{$\begin{array}{l}\text { Independent variables } \\
\text { entered }\end{array}$} & \multicolumn{6}{|l|}{ Statistics for best model } \\
\hline & & & $\begin{array}{l}\text { Predictors retained, } \\
\text { standardized } \beta \text { values }\end{array}$ & $R^{2}$ & $\operatorname{Adj} . R^{2}$ & AlCc & $\operatorname{AICC}^{a}(n=15)$ & $F(\mathrm{df})$ \\
\hline \multirow[t]{3}{*}{ 2-back AHR } & $\begin{array}{l}\text { Load-dependent } \\
\quad \text { connectivity }(n=39)\end{array}$ & 6 network pairs & IFPN-rFPN, $\beta=0.55^{* * *}$ & 0.30 & 0.28 & -133 & -61.3 & $15.7^{* * *}(1,38)$ \\
\hline & $\begin{array}{l}\text { Load-independent } \\
\quad \text { connectivity }(n=39)\end{array}$ & 6 network pairs & CON-rFPN $\beta=-0.22$ & 0.05 & 0.02 & -121 & -44.5 & $1.8(1,38)$ \\
\hline & $\begin{array}{l}\text { Load-dependent } \\
\quad \text { activation }(n=39)\end{array}$ & 4 networks & CON,$\beta=-0.47^{*}$ IFPN, $\beta=0.52^{* *}$ & 0.22 & 0.18 & -125 & -43.0 & $5.1^{*}(2,38)$ \\
\hline \multirow[t]{3}{*}{$\begin{array}{l}\text { Differential accuracy } \\
\qquad(2 \text {-back - 1-back) }\end{array}$} & $\begin{array}{l}\text { Load-dependent } \\
\quad \text { connectivity }(n=39)\end{array}$ & 6 network pairs & IFPN-rFPN, $\beta=0.39^{*}$ & 0.15 & 0.13 & -128 & -40.9 & $6.7^{*}(1,38)$ \\
\hline & $\begin{array}{l}\text { Load-independent } \\
\quad \text { connectivity }(n=39)\end{array}$ & 6 network pairs & CON-IFPN $\beta=0.22$ & 0.05 & 0.02 & -124 & -42.6 & $1.8(1,38)$ \\
\hline & $\begin{array}{l}\text { Load-dependent } \\
\text { activation }(n=39)\end{array}$ & 4 networks & IFPN, $\beta=0.47^{*}$ rFPN, $\beta=-0.45$ & 0.12 & 0.07 & -123 & -35.0 & $2.5(2,38)$ \\
\hline
\end{tabular}

${ }^{a}$ AIC, Corrected AIC calculated to compare models on the subsample with PET dopamine measures ( $\left.n=15\right)$.

${ }^{* * *} p<0.001,{ }^{* *} p<0.01,{ }^{*} p<0.05$.

\section{A}

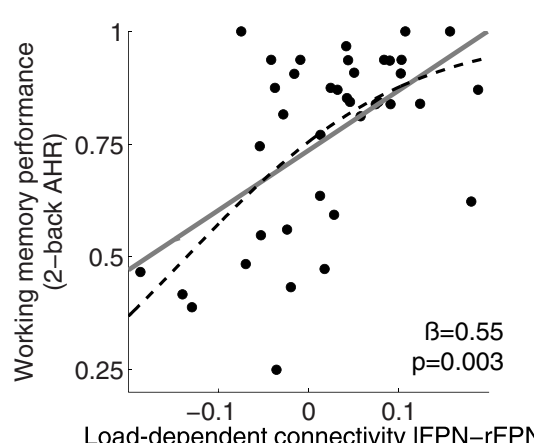

D

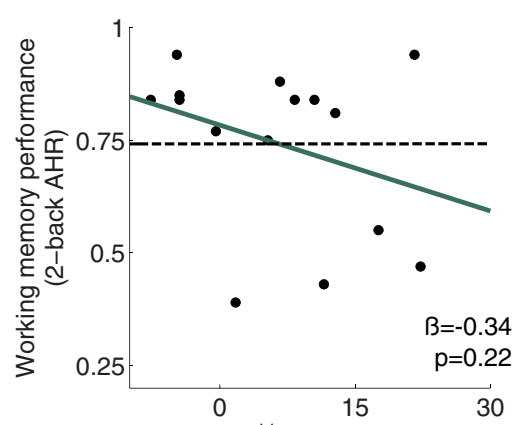

Displacement $\left[{ }^{11} \mathrm{C}\right] \mathrm{FLB} 457$, DLPFC (\%)
B

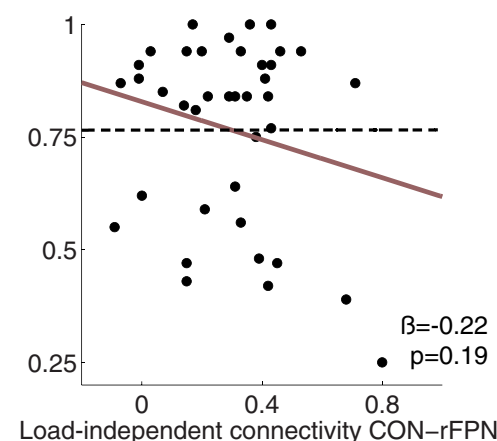

Load-independent connectivity CON-rFPN

\section{C}

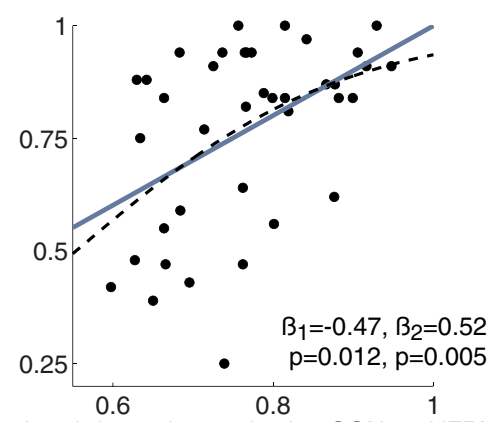

Load-dependent activation CON and IFPN (predicted value)

E

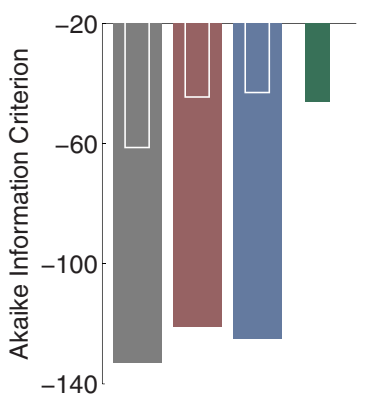

Load-dependent connectivity Load-independent connectivity Load-dependent activation Dopamine release capacity

Figure 3. $\boldsymbol{A}-\boldsymbol{D}$, Scatterplots indicating the relationship between working memory performance and different imaging measures in healthy individuals ( $n=39$ ). Solid lines represent linear fits and broken lines represent logistic fits. For each type of imaging measure, the best predictor or set of predictors were selected. The model in $C$ includes two predictor variables and statistics are presented for both of them ( $\beta_{1}=$ CON; $\beta_{2}=$ IFPN). $E$, Comparison of regression models presented in the scatterplots. According to both AIC and adjusted $R^{2}$, load-dependent connectivity was the imaging measure which best predicted working memory performance This was also true if logistic fits were used (data not shown). AIC is shown for models including the full sample (broad bars) as well for models including only the subsample with data for dopamine-release capacity (inset, narrow bars).

$\Delta$ connectivity, lFPN-rFPN showed that the load-dependent activations did not significantly explain any further variance in task performance than that explained by the load-dependent connectivity measure alone. Last, although load-dependent activation of the IFPN and CON predicted task performance when included in a regression model together, neither IC significantly predicted task performance when included alone in univariate regression (CON: standardized $\beta=-0.18, p=0.28$; IFPN: standardized $\beta=0.27, p=0.10$ ), further reinforcing the notion that it is the interplay between networks rather than activity in a single network which is most predictive of interindividual variability in working memory performance. Thus, these results suggest that load-dependent connectivity between working-memory networks is a better predictor of working memory performance than other fMRI and PET measures that are more commonly used. 
Role of dopamine release capacity in load-dependent internetwork connectivity

Contrary to our hypothesis, DLPFC dopamine release and $\mathrm{D} 2$ receptor availability did not predict $\Delta$ connectivity 1 FPN-rFPN when included together in a linear regression model (model: $F_{(2,14)}=1.9, p=0.19$, $R^{2}=0.24 ; \Delta \mathrm{BP}_{\mathrm{ND}}$ : standardized $\beta=$ $-0.71, p=0.077 ; \mathrm{BP}_{\mathrm{ND}}$ : standardized $\beta=$ $0.59, p=0.13$ ) or when included alone in separate models (all $p$ values $>0.3$ ). Thus, load-dependent connectivity between networks did not seem to relate to dopamine signaling, at least not to the PET dopamine measures we obtained in vivo.

\section{Study 2 (healthy individuals compared} with patients with schizophrenia)

We next investigated the clinical relevance of load-dependent internetwork connectivity by comparing a subsample of these healthy participants to 15 unmedicated patients with schizophrenia. Consistent with the well established deficit in working memory associated with this disorder (Lee and Park, 2005), the schizophrenia group showed a large deficit relative to the whole group of healthy controls in working memory performance according to the 2-back AHR (two-sample $t$ test, $t_{(52)}=$ 2.7, $p=0.010$, Cohen's $d=0.74$ ) but this was not the case when comparing against the subsample of healthy participants matched at the group level on working memory performance, demographics, and fMRI scan quality (all $p$ values $>0.4$ ); see Materials and Methods; Table 1).

We first aimed to confirm that patients and healthy controls both used the same working-memory networks by searching for networks showing significant load-dependent activation in patients. Similar to controls, patients with schizophrenia showed significant load-dependent increase in activation of the lFPN and rFPN and decrease in activation of the aDMN (all $p$ values $<0.05$ ); however, patients did not show evidence of loaddependent activation of the CON (one-sample $t$ test, $p=0.31$ ). Aside from these three ICs, there was little indication of loaddependent activation of any other ICs in schizophrenia (all other ICs had one-sample $t$ values $<1.6$ ). There were no significant differences in load-dependent activation of any of these networks between the 2 groups (all $p$ values $>0.7$ ).

We sought to confirm that any group differences related to internetwork connectivity would not be secondary to differences in the structure of the networks themselves by examining intranetwork connectivity as measured from the ICA-derived spatial maps which reflect the weight of each voxel on each component. We compared subject-level connectivity maps for the four networks between unmedicated schizophrenia patients and matched controls via voxelwise, two-sample $t$ tests. Although previous studies have shown abnormalities in intranetwork connectivity in schizophrenia (Jafri et al., 2008; Lui et al., 2010; van den Heuvel and Fornito, 2014) we found no significant differ-
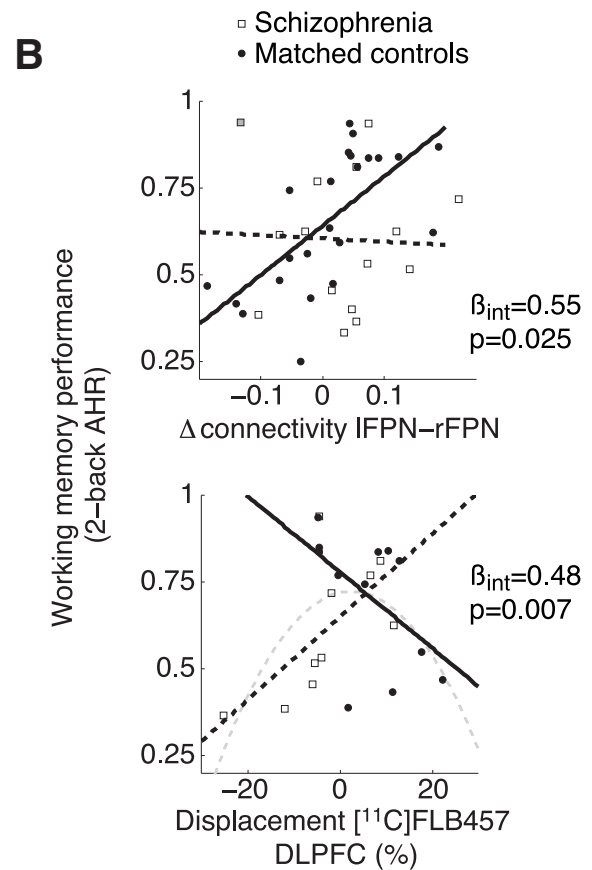

Schizophrenia

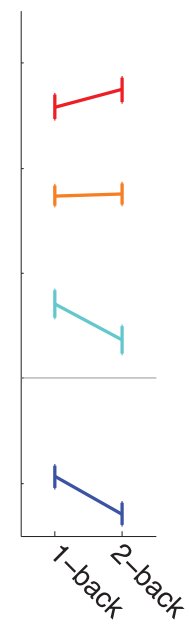

rFPN-CON rFPN-aDMN

Figure 4. Comparing individuals with schizophrenia to matched healthy controls. $A$, Load-dependent changes in internetwork

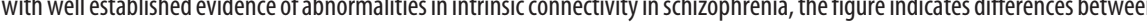

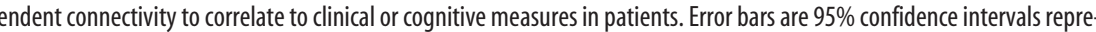
(cous $\beta=-0.04, p=0.86$ ). There was evidence that one patient (indicated by gray-filled square in top-left corner) was an influential combining the groups together, the relationship between these variables fit an inverted-U-shaped curve (broken gray line). $\beta_{\text {int }}$ Standardized $\beta$ for the interaction term of imaging measure by group.

ences between groups for any of the 4 maps (all cluster-level FWE-corrected $p$ values $>0.05$ ).

The schizophrenia group did not differ significantly from matched controls on load-dependent internetwork connectivity for any of the six IC pairs (two-sample tests, all $p$ values $>0.17$; Fig. 4A). Unlike healthy controls, a backward stepwise linear regression in patients to predict 2-back AHR based on loaddependent connectivity did not retain any network pairs. To investigate whether the relationship between working memory performance and $\Delta$ connectivity lFPN-rFPN was different in schizophrenia compared with healthy controls, we conducted a linear regression analysis with 2-back AHR as dependent variable and three independent variables: group, $\Delta$ connectivity IFPN$\mathrm{rFPN}$, and an interaction term of group by $\Delta$ connectivity lFPN$\mathrm{rFPN}$. We found a significant interaction (standardized $\beta=0.55$, $p=0.025$ ) indicating that working memory performance was more closely related to $\Delta$ connectivity lFPN-rFPN in controls than in patients, for whom these factors appeared unrelated (in patients $\Delta$ connectivity lFPN-rFPN standardized $\beta=-0.04, p=$ 0.86; Fig. $4 B$ ). We found evidence that one patient could be an influential outlier in this regression (Cook's distance $>4 / n$; Fig. $4 B)$. Therefore we reran the analysis using robust regression 
(robustfit in MATLAB) and the group by $\Delta$ connectivity lFPNrFPN interaction was still significant (standardized $\beta=0.52, p=$ 0.045 ) and the slope relating $\Delta$ connectivity lFPN-rFPN to performance remained flat in patients (standardized $\beta=-0.003$, $p=0.99$ ). To further ensure this result was not due to the spatial structure of the networks differing between the groups we ran separate ICAs for patients and controls and found that the relationship between $\Delta$ connectivity lFPN-rFPN and task performance for the respective groups remained similar (controls: standardized $\beta=0.66$; patients: standardized $\beta=-0.18$ ) to those from the combined ICA including all subjects described above. Thus, although most patients with schizophrenia showed the expected changes in FPN connectivity in response to working memory load, this increase was not related to intersubject variation in working memory performance.

Furthermore, the significant group by $\Delta$ connectivity lFPNrFPN interaction on working memory performance was not likely due to abnormalities in DLPFC dopamine release capacity in patients. An exploratory analysis $(n=10$ schizophrenia patients and 12 healthy controls) using the above regression model with the addition of DLPFC dopamine release and an interaction term for DLPFC dopamine release by $\Delta$ connectivity lFPN-rFPN found that the interaction of group by $\Delta$ connectivity lFPN-rFPN remained nearly significant despite the decrease in degrees of freedom in this analysis (standardized $\beta=0.78, p=0.062$ ).

Because our principal measure of interest, $\Delta$ connectivity IFPN-rFPN, did not predict interindividual differences in task performance in patients, we performed further exploratory analyses in search for a variable that would explain variability in performance among patients. Given interest in the role of cortical dopamine in working memory deficits in schizophrenia (Weinberger et al., 1988; Durstewitz and Seamans, 2008; Rolls et al., 2008; Arnsten and Jin, 2014), we investigated whether patients' performance might be related to PET dopamine measures, despite failing to detect such a relationship in healthy individuals. We found that a backward stepwise regression including DLPFC $\mathrm{BP}_{\mathrm{ND}}$ and $\Delta \mathrm{BP}_{\mathrm{ND}}$ retained $\Delta \mathrm{BP}_{\mathrm{ND}}$ as a significant predictor of working memory performance $\left(R^{2}=0.41\right.$, standardized $\beta=$ $0.64, p=0.046$; Fig. 4 ) indicating that patients with lower dopamine release capacity had poorer working memory performance. We next tested patients and matched controls together in a linear regression analysis with 2-back AHR as dependent variable and three independent variables: group, DLPFC $\triangle \mathrm{BP}_{\mathrm{ND}}$, and an interaction term of group by DLPFC $\triangle \mathrm{BP}_{\mathrm{ND}}$. We found a significant interaction $\left(R^{2}=0.38\right.$, standardized $\left.\beta=0.86, p=0.007\right)$, indicating that the effect of dopamine release capacity on performance was significantly more positive in patients than controls (Fig. 4). Similar to our previous report of an overlapping cohort (Slifstein et al., 2015), patients in this study had lower DLPFC dopamine release than controls $\left(\mathrm{t}_{21}=-2.3, p=0.035\right)$. This fact, combined with the difference in slopes between patients and controls led us to test a quadratic model (inverted $\mathrm{U}$ ) with the groups combined to predict performance based on $\triangle \mathrm{BP}_{\mathrm{ND}}$ and $\left(\Delta \mathrm{BP}_{\mathrm{ND}}\right)^{2}$. Although the overall model was not significant $\left(F_{(2,21)}=3.2, p=0.061\right)$, the quadratic term was a significant predictor (standardized $\beta=-0.48, p=0.025$ ), and the Akaike information criterion (AIC) for the quadratic model was similar to that of the linear model where separate slopes were fit for each group (both AIC $=-69$ ). Together, this provides some support for the notion of an optimal range for dopamine release outside of which performance drops. In this framework, patients in general would occupy the lower end of this range and those with more dopamine release capacity would be closer to the optimal range and would thus have better performance. Altogether, these exploratory analyses could suggest that dynamic connectivity and dopamine may be affecting working memory performance through different pathways with the former being more relevant in controls and the latter in patients.

There were no significant relationships between $\Delta$ connectivity lFPN-rFPN and dopamine measures in patients.

Finally, exploratory analyses in a subset of 12 patients with available clinical severity scores showed a significant relationship between $\Delta$ connectivity IFPN-rFPN and positive symptoms (SAPS: standardized $\beta=-0.66, p=0.020$ ) but not negative symptoms (SANS: standardized $\beta=0.10, p=0.75$ ); indicating patients with higher positive symptom scores had lower $\Delta$ connectivity lFPN-rFPN.

\section{Discussion}

Our findings provide evidence that dynamic interactions between brain networks underlie successful working memory performance. A novel fMRI measure of working memory loaddependent internetwork connectivity predicted interindividual differences in task performance better than other imaging measures, suggesting that brain-network dynamics may be a key factor in determining adaptive, goal-oriented behaviors. We did not find evidence that adaptive communication between brain networks could be a mechanism through which dopamine signaling modulates cognitive performance. Adaptive internetwork communication is observed in schizophrenia but may not effectively subserve working memory in this condition. We found preliminary evidence that deficient prefrontal dopamine release could directly underlie interindividual differences in working memory performance among patients with schizophrenia.

\section{Load-dependent internetwork connectivity}

Previous studies of working memory have investigated connectivity of brain networks (Meda et al., 2009; Sambataro et al., 2009) or task-dependent changes in connectivity between specific brain regions (Anticevic et al., 2010, 2012; Rasetti et al., 2011;Bähner et al., 2015). Our study is to our knowledge the first combining both approaches and investigating loaddependent internetwork connectivity during working memory. Consistent with our findings, previous studies found working memory to be subserved by frontoparietal networks (Kim et al., 2009; Meda et al., 2009; Sambataro et al., 2009) and prefrontal interhemispheric connectivity to increase with working memory load (Rasetti et al., 2011).

Load-dependent connectivity was a better predictor of working memory performance across subjects than load-dependent activation, load-independent connectivity, or PET dopamine measures, suggesting that it is the capacity for the networks to dynamically adjust connectivity in response to changes in cognitive demand that is essential for successful execution of complex cognitive processes like working memory. Furthermore, loaddependent connectivity suffers less from limitations inherent to the other fMRI measures which may obscure associations with task performance: it is hard to disentangle whether low loaddependent activation would be associated with poor performance (eg, due to task disengagement) or good performance (eg, indicative of less error-related activation (Danielmeier et al., 2011), greater metabolic efficiency, or less time on task). On the other hand, load-independent connectivity does not take into account how neural function is influenced by task manipulations thereby overlooking critical information, which is captured by load-dependent connectivity. 
Our data suggest that increased communication between frontoparietal networks accounts for the association between load-dependent connectivity and working memory performance because increased load-dependent activity in both networks (which could spuriously inflate the "connectivity" measured between them even if they act in parallel) did not predict performance. This increase in internetwork communication may reflect enhanced integration of distinct functions subserved by each of these networks (Goel, 2015). Increased connectivity between FPNs could also be due to a shift in network structure toward a merged FPN spanning both hemispheres; however, an exploratory analysis performing ICA separately in 1-back and 2-back conditions revealed similar network structure in the two conditions (data not shown).

\section{Dopamine, load-dependent internetwork connectivity, and working memory}

Our hypothesis that load-dependent connectivity between workingmemory networks would be related to cortical dopamine release capacity across subjects was not confirmed. Because this relationship was not significant for the fMRI measure we have shown to be most related to working memory performance, it raises the question of whether the role of dopamine release on working memory is independent of network-level brain processing. Nevertheless, we do not view this result as conclusive and further replication in a larger sample is warranted; especially in light of the nonsignificant trend we observed relating dopamine and dynamic connectivity measures. Furthermore, our PET dopamine measure may not be ideal for our purposes because we examine amphetamine-induced dopamine release rather than task-induced release. Task-induced cortical DA release, although preferable, has not been shown to be a reliable measure with PET methodology.

Studies looking at effects of dopaminergic challenges (Sawaguchi and Goldman-Rakic, 1991) or genetic variation (Sambataro et al., 2009) have indicated a critical role for cortical dopamine release in working memory. However, in healthy individuals we did not find evidence supporting a role for cortical dopamine release capacity in explaining interindividual variability in working memory performance. This inconsistency may be due to limitations in power or the type of dopamine measure used (described in the preceding paragraph) or else it could be due to difficulties predicting the impact of dopamine function on cognition given the inverted-U function governing their relationship (Cools and D'Esposito, 2011).

\section{Load-dependent internetwork connectivity and schizophrenia}

Our main finding in the clinical sample was that the seemingly normal changes in load-dependent connectivity between FPNs shown by unmedicated individuals with schizophrenia did not predict better task performance whereas deficits in dopamine release capacity did. Both of these relationships were in contrast to what we observed in controls. Schizophrenia is associated with deficits in working memory and other aspects of cognition (Lee and Park, 2005) and has long been theorized to be a disorder of dysconnectivity (Stephan et al., 2009). The neurobiological basis of working memory deficits in schizophrenia remains poorly understood and it is difficult to disentangle effects primary to the disorder from those secondary to treatment with antipsychotic medication, a confound minimized in our unmedicated sample. Past research has indicated that working memory deficits in this disorder could be related to abnormal connectivity between taskpositive and task-negative networks (Whitfield-Gabrieli et al.,
2009), whereas connectivity within and between the DMN, CON, and FPN have all been implicated in schizophrenia (Jafri et al., 2008; Whitfield-Gabrieli et al., 2009; Lui et al., 2010; Manoliu et al., 2014; Pankow et al., 2015). However, our study is the first to investigate the role of task-dependent modulation of internetwork connectivity in the cognitive deficits of schizophrenia.

It is intriguing that the main factor explaining interindividual differences in working memory performance in health was unrelated to performance in unmedicated individuals with schizophrenia, suggesting that patients' brains may not be properly exploiting these dynamic network interactions. The deficit could lie downstream in cognitive processing from the increase in FPN connectivity or else different networks could usurp working memory functions in schizophrenia (although we did not see evidence of this). It is also possible that the deficit in loaddependent connectivity in schizophrenia could not be detected by the large-scale spatial and temporal resolution of our measure. Indeed, a previous report found individuals with schizophrenia to have decreased working memory-load-dependent connectivity between left and right prefrontal cortex (Rasetti et al., 2011). The absence of a similar deficit in load-dependent connectivity between frontoparietal networks (or other working memoryrelated networks) should be interpreted with caution since our analyses may have lacked sufficient statistical power to detect it.

Our exploratory analysis did not find evidence to support the possibility that dynamic connectivity fails to influence working memory performance in schizophrenia patients due to deficits in cortical dopamine release capacity in this condition (Slifstein et al., 2015). However, cortical dopamine release deficits themselves predicted poor performance in schizophrenia, consistent with long-theorized ideas about dopamine and cognition in schizophrenia (Rolls et al., 2008; Weinberger et al., 1988). Furthermore, the relationship between dopamine release and working memory performance had an opposite linear trend in patients and controls and, when combining the 2 groups, showed some evidence of an inverted-U pattern. Thus for individuals with schizophrenia who tend to have deficits in dopamine release, higher release capacity relates to better performance. Lack of adequate dopamine release may impair D1-receptor-mediated recurrent excitation within neural networks that maintain representations held in memory and thereby decrease their signal-to-noise ratio and produce less stable attractor states (Durstewitz and Seamans, 2002; Rolls et al., 2008). It is also possible that increased dopamine release may only be helpful for individuals with poor working memory, whereas higher performers may have sufficient cognitive resources or strategies to make them less dependent on dopamine. Although our findings relating dopamine release to working memory are interesting, they were the result of exploratory analyses and not the primary focus of the current study. They should be interpreted with caution because of the limited statistical power and our failure to observe a similar relationship in a larger study using other working-memory tasks (Slifstein et al., 2015).

Despite not relating to patients' task performance, we were intrigued to observe that our principal fMRI measure of interest, $\Delta$ connectivity lFPN-rFPN correlated with severity of positive symptoms. This observation is consistent with the theory that positive symptoms are related to noisy signaling in cortical networks (Rolls et al., 2008), which could impede load-dependent changes in their functional connectivity. However, due to the small sample, this finding should be considered with caution and warrants replication. 


\section{Conclusion}

We found that connectivity between brain networks changes as a function of cognitive load during a working-memory task. This measure of load-dependent connectivity outperformed traditional fMRI and PET measures of dopamine release capacity in predicting brain-behavior relationships in healthy individuals. Such dynamic interactions of brain systems appear to be relevant to understanding cognitive deficits in psychiatric conditions since we observed that the relationship between load-dependent network connectivity and task performance was disrupted in patients with schizophrenia. Together, our findings suggest that dynamic interactions between brain networks may support the type of flexible adaptations in response to changing environmental demands that are essential to our everyday functioning.

\section{References}

Abi-Dargham A, Martinez D, Mawlawi O, Simpson N, Hwang DR, Slifstein M, Anjilvel S, Pidcock J, Guo NN, Lombardo I, Mann JJ, Van Heertum R, Foged C, Halldin C, Laruelle M (2000) Measurement of striatal and extrastriatal dopamine D1 receptor binding potential with [11C]NNC 112 in humans: validation and reproducibility. J Cereb Blood Flow Metab 20:225-243. CrossRef Medline

Abi-Dargham A, Xu X, Thompson JL, Gil R, Kegeles LS, Urban N, Narendran R, Hwang DR, Laruelle M, Slifstein M (2012) Increased prefrontal cortical $D(1)$ receptors in drug naive patients with schizophrenia: a PET study with $\left[{ }^{11} \mathrm{C}\right] \mathrm{NNC112}$. J Psychopharmacol 26:794-805. CrossRef Medline

Allen EA, Erhardt EB, Damaraju E, Gruner W, Segall JM, Silva RF, Havlicek M, Rachakonda S, Fries J, Kalyanam R, Michael AM, Caprihan A, Turner JA, Eichele T, Adelsheim S, Bryan AD, Bustillo J, Clark VP, Feldstein Ewing SW, Filbey F, et al. (2011) A baseline for the multivariate comparison of resting-state networks. Front Syst Neurosci 5:2. Medline

Andreasen NC (1983) Scale for the assessment of negative symptoms (SANS). Iowa city, IA: University of Iowa.

Andreasen NC (1984) Scale for the assessment of positive symptoms (SAPS). Iowa city, IA: University of Iowa.

Anticevic A, Repovs G, Shulman GL, Barch DM (2010) When less is more: TPJ and default network deactivation during encoding predicts working memory performance. Neuroimage 49:2638-2648. CrossRef Medline

Anticevic A, Repovs G, Krystal JH, Barch DM (2012) A broken filter: prefrontal functional connectivity abnormalities in schizophrenia during working memory interference. Schizophr Res 141:8-14. CrossRef Medline

Arnsten AF, Jin LE (2014) Molecular influences on working memory circuits in dorsolateral prefrontal cortex. Prog Mol Biol Transl Sci 122: 211-231. CrossRef Medline

Bähner F, Demanuele C, Schweiger J, Gerchen MF, Zamoscik V, Ueltzhöffer K, Hahn T, Meyer P, Flor H, Durstewitz D, Tost H, Kirsch P, Plichta MM, MeyerLindenberg A (2015) Hippocampal-dorsolateral prefrontal coupling as a species-conserved cognitive mechanism: a human translational imaging study. Neuropsychopharmacology 40:1674-1681. CrossRef Medline

Balsters JH, Robertson IH, Calhoun VD (2013) BOLD frequency power indexes working memory performance. Front Hum Neurosci 7:207. CrossRef Medline

Baron RM, Kenny DA (1986) The moderator-mediator variable distinction in social psychological research: conceptual, strategic, and statistical considerations. J Pers Soc Psychol 51:1173-1182. CrossRef Medline

Bell AJ, Sejnowski TJ (1995) An information-maximization approach to blind separation and blind deconvolution. Neural Comput 7:1129-1159. CrossRef Medline

Bray S, Arnold AE, Levy RM, Iaria G (2015) Spatial and temporal functional connectivity changes between resting and attentive states. Hum Brain Mapp 36:549-565. CrossRef Medline

Calhoun VD, Maciejewski PK, Pearlson GD, Kiehl KA (2008) Temporal lobe and "default" hemodynamic brain modes discriminate between schizophrenia and bipolar disorder. Hum Brain Mapp 29:1265-1275. CrossRef Medline

Cole DM, Oei NY, Soeter RP, Both S, van Gerven JM, Rombouts SA, Beckmann CF (2013) Dopamine-dependent architecture of cortico-subco- rtical network connectivity. Cereb Cortex 23:1509-1516. CrossRef Medline

Cools R, D'Esposito M (2011) Inverted-U-shaped dopamine actions on human working memory and cognitive control. Biol Psychiatry 69:e113-25. CrossRef Medline

Cousineau D (2005) Confidence intervals in within-subjects designs: a simpler solution to Loftus and Masson's method. TQMP 1:42-45.

Curtis CE, Zald DH, Pardo JV (2000) Organization of working memory within the human prefrontal cortex: a PET study of self-ordered object working memory. Neuropsychologia 38:1503-1510. CrossRef Medline

Damoiseaux JS, Rombouts SA, Barkhof F, Scheltens P, Stam CJ, Smith SM, Beckmann CF (2006) Consistent resting-state networks across healthy subjects. Proc Natl Acad Sci U S A 103:13848-13853. CrossRef Medline

Dang LC, O’Neil JP, Jagust WJ (2012) Dopamine supports coupling of attention-related networks. J Neurosci 32:9582-9587. CrossRef Medline

Danielmeier C, Eichele T, Forstmann BU, Tittgemeyer M, Ullsperger M (2011) Posterior medial frontal cortex activity predicts post-error adaptations in task-related visual and motor areas. J Neurosci 31:1780-1789. CrossRef Medline

Durstewitz D, Seamans JK (2002) The computational role of dopamine D1 receptors in working memory. Neural Netw 15:561-572. CrossRef Medline

Durstewitz D, Seamans JK (2008) The dual-state theory of prefrontal cortex dopamine function with relevance to catechol-o-methyltransferase genotypes and schizophrenia. Biol Psychiatry 64:739-749. CrossRef Medline

Eichele T, Debener S, Calhoun VD, Specht K, Engel AK, Hugdahl K, von Cramon DY, Ullsperger M (2008) Prediction of human errors by maladaptive changes in event-related brain networks. Proc Natl Acad Sci U S A 105:6173-6178. CrossRef Medline

First M, Spitzer R, Gibbon M,Williams J (1995) Structured Clinical Interview for DSM-IV Axis I Disorders (SCID-I/P, Version 2.0). Biometrics Research Department. New York: New York State Psychiatric Institute.

Fox MD, Snyder AZ, Vincent JL, Corbetta M, Van Essen DC, Raichle ME (2005) The human brain is intrinsically organized into dynamic, anticorrelated functional networks. Proc Natl Acad Sci U S A 102:9673-9678. CrossRef Medline

Freire L, Roche A, Mangin JF (2002) What is the best similarity measure for motion correction in fMRI time series? IEEE Trans Med Imaging 21: 470-484. CrossRef Medline

Friston KJ, Buechel C, Fink GR, Morris J, Rolls E, Dolan RJ (1997) Psychophysiological and modulatory interactions in neuroimaging. Neuroimage 6:218-229. CrossRef Medline

Goel V (2015) Indeterminacy tolerance as a basis of hemispheric asymmetry within prefrontal cortex. Front Hum Neurosci 9:326. CrossRef Medline

Haatveit BC, Sundet K, Hugdahl K, Ueland T, Melle I, Andreassen OA (2010) The validity of $d$ prime as a working memory index: results from the "Bergen $n$-back task." J Clin Exp Neuropsychol 32:871-880. CrossRef Medline

Hollingshead AB (1975) Four factor index of social status. New Haven, Connecticut: Working paper published by the author.

Hutchison RM, Womelsdorf T, Allen EA, Bandettini PA, Calhoun VD, Corbetta M, Della Penna S, Duyn JH, Glover GH, Gonzalez-Castillo J, Handwerker DA, Keilholz S, Kiviniemi V, Leopold DA, de Pasquale F, Sporns O, Walter M, Chang C (2013) Dynamic functional connectivity: promise, issues, and interpretations. Neuroimage 80:360-378. CrossRef Medline

Jafri MJ, Pearlson GD, Stevens M, Calhoun VD (2008) A method for functional network connectivity among spatially independent resting-state components in schizophrenia. Neuroimage 39:1666-1681. CrossRef Medline

Kelly C, de Zubicaray G, Di Martino A, Copland DA, Reiss PT, Klein DF, Castellanos FX, Milham MP, McMahon K (2009) L-Dopa modulates functional connectivity in striatal cognitive and motor networks: a double-blind placebo-controlled study. J Neurosci 29:7364-7378. CrossRef Medline

Kim DI, Manoach DS, Mathalon DH, Turner JA, Mannell M, Brown GG, Ford JM, Gollub RL, White T, Wible C, Belger A, Bockholt HJ, Clark VP, Lauriello J, O'Leary D, Mueller BA, Lim KO, Andreasen N, Potkin SG, Calhoun VD (2009) Dysregulation of working memory and defaultmode networks in schizophrenia using independent component analysis, an fBIRN and MCIC study. Hum Brain Mapp 30:3795-3811. CrossRef Medline 
Lee J, Park S (2005) Working memory impairments in schizophrenia: a meta-analysis. J Abnorm Psychol 114:599-611. CrossRef Medline

Li YO, Adali T, Calhoun VD (2007) Estimating the number of independent components for functional magnetic resonance imaging data. Hum Brain Mapp 28:1251-1266. CrossRef Medline

Linden DE (2007) The working memory networks of the human brain. Neuroscientist 13:257-267. CrossRef Medline

Lui S, Li T, Deng W, Jiang L, Wu Q, Tang H, Yue Q, Huang X, Chan RC, Collier DA, Meda SA, Pearlson G, Mechelli A, Sweeney JA, Gong Q (2010) Short-term effects of antipsychotic treatment on cerebral function in drug-naive first-episode schizophrenia revealed by "resting state" functional magnetic resonance imaging. Arch Gen Psychiatry 67: 783-792. CrossRef Medline

MacMillan NA, CD (2005) Detection theory: a user's guide. London, UK: Lawrence Erlbaum Associates.

Manoliu A, Riedl V, Zherdin A, Muhlau M, Schwerthöffer D, Scherr M, Peters H, Zimmer C, Förstl H, Bäuml J, Wohlschläger AM, Sorg C (2014) Aberrant dependence of default mode/central executive network interactions on anterior insular salience network activity in schizophrenia. Schizophr Bull 40:428-437. CrossRef Medline

Meda SA, Stevens MC, Folley BS, Calhoun VD, Pearlson GD (2009) Evidence for anomalous network connectivity during working memory encoding in schizophrenia: an ICA based analysis. PLoS One 4:e7911. CrossRef Medline

Nagano-Saito A, Leyton M, Monchi O, Goldberg YK, He Y, Dagher A (2008) Dopamine depletion impairs frontostriatal functional connectivity during a set-shifting task. J Neurosci 28:3697-3706. CrossRef Medline

Narendran R, Frankle WG, Mason NS, Muldoon MF, Moghaddam B (2012) Improved working memory but no effect on striatal vesicular monoamine transporter type 2 after omega- 3 polyunsaturated fatty acid supplementation. PLoS One 7:e46832. CrossRef Medline

Nurnberger JI Jr, Blehar MC, Kaufmann CA, York-Cooler C, Simpson SG, Harkavy-Friedman J, Severe JB, Malaspina D, Reich T. Diagnostic interview for genetic studies. Rationale, unique features, and training. NIMH Genetics Initiative. Arch Gen Psychiatry 51:849-859, 1994; discussion 863-864.

Oldfield RC (1971) The assessment and analysis of handedness: the Edinburgh inventory. Neuropsychologia 9:97-113. CrossRef Medline

Pankow A, Deserno L, Walter M, Fydrich T, Bermpohl F, Schlagenhauf F, Heinz A (2015) Reduced default mode network connectivity in schizophrenia patients. Schizophr Res 165:90-93. CrossRef Medline

Parnaudeau S, O'Neill PK, Bolkan SS, Ward RD, Abbas AI, Roth BL, Balsam PD, Gordon JA, Kellendonk C (2013) Inhibition of mediodorsal thalamus disrupts thalamofrontal connectivity and cognition. Neuron 77: 1151-1162. CrossRef Medline

Power JD, Barnes KA, Snyder AZ, Schlaggar BL, Petersen SE (2012) Spurious but systematic correlations in functional connectivity MRI networks arise from subject motion. Neuroimage 59:2142-2154. CrossRef Medline

Rasetti R, Sambataro F, Chen Q, Callicott JH, Mattay VS, Weinberger DR (2011) Altered cortical network dynamics: a potential intermediate phenotype for schizophrenia and association with ZNF804A. Arch Gen Psychiatry 68:1207-1217. CrossRef Medline

Rolls ET, Loh M, Deco G, Winterer G (2008) Computational models of schizophrenia and dopamine modulation in the prefrontal cortex. Nat Rev Neurosci 9:696-709. CrossRef Medline

Saliasi E, Geerligs L, Lorist MM, Maurits NM (2014) Neural correlates associated with successful working memory performance in older adults as revealed by spatial ICA. PLoS One 9:e99250. CrossRef Medline

Sambataro F, Reed JD, Murty VP, Das S, Tan HY, Callicott JH, Weinberger DR, Mattay VS (2009) Catechol-O-methyltransferase valine (158) methionine polymorphism modulates brain networks underlying working memory across adulthood. Biol Psychiatry 66:540-548. CrossRef Medline

Sawaguchi T, Goldman-Rakic PS (1991) D1 dopamine receptors in prefrontal cortex: involvement in working memory. Science 251:947-950. CrossRef Medline

Shen K, Hutchison RM, Bezgin G, Everling S, McIntosh AR (2015) Network structure shapes spontaneous functional connectivity dynamics. J Neurosci 35:5579-5588. CrossRef Medline

Slifstein M, van de Giessen E, Van Snellenberg J, Thompson JL, Narendran R, Gil R, Hackett E, Girgis R, Ojeil N, Moore H, D'Souza D, Malison RT, Huang Y, Lim K, Nabulsi N, Carson RE, Lieberman JA, Abi-Dargham A (2015) Deficits in prefrontal cortical and extrastriatal dopamine release in schizophrenia: a positron emission tomographic functional magnetic resonance imaging study. JAMA Psychiatry 72:316-324. CrossRef Medline

Stephan KE, Friston KJ, Frith CD (2009) Dysconnection in schizophrenia: from abnormal synaptic plasticity to failures of self-monitoring. Schizophr Bull 35:509-527. CrossRef Medline

Terán-Pérez GR, González-Robles RO, Tarrago-Castellanos R, Mercadillo RE, Jiménez-Anguiano A, Velázquez-Moctezuma J (2012) Sleep deprivation affects working memory in low but not in high complexity for the $n$-back test. Neurosci Med 3:380-386. CrossRef

Thompson GJ, Magnuson ME, Merritt MD, Schwarb H, Pan WJ, McKinley A, Tripp LD, Schumacher EH, Keilholz SD (2013) Short-time windows of correlation between large-scale functional brain networks predict vigilance intraindividually and interindividually. Hum Brain Mapp 34: 3280-3298. CrossRef Medline

van den Heuvel MP, Fornito A (2014) Brain networks in schizophrenia. Neuropsychol Rev 24:32-48. CrossRef Medline

Van Snellenberg JX, Slifstein M, Read C, Weber J, Thompson JL, Wager TD, Shohamy D, Abi-Dargham A, Smith EE (2015) Dynamic shifts in brain network activation during supracapacity working memory task performance. Hum Brain Mapp 36:1245-1264. CrossRef Medline

Wager TD, Waugh CE, Lindquist M, Noll DC, Fredrickson BL, Taylor SF (2009) Brain mediators of cardiovascular responses to social threat: part I: reciprocal dorsal and ventral sub-regions of the medial prefrontal cortex and heart-rate reactivity. Neuroimage 47:821-835. CrossRef Medline

Weinberger DR, Berman KF, Chase TN (1988) Mesocortical dopaminergic function and human cognition. Ann N Y Acad Sci 537:330-338. CrossRef Medline

Whitfield-Gabrieli S, Thermenos HW, Milanovic S, Tsuang MT, Faraone SV, McCarley RW, Shenton ME, Green AI, Nieto-Castanon A, LaViolette P, Woicik J, Gabrieli JD, Seidman LJ (2009) Hyperactivity and hyperconnectivity of the default network in schizophrenia and in first-degree relatives of persons with schizophrenia. Proc Natl Acad Sci U S A 106: 1279-1284. CrossRef Medline 